\title{
MICRORNAS AS NON-INVASIVE SCREENING BIOMARKERS
} OF COLORECTAL CANCER

\author{
ROBERTA MARIA MANZAT SAPLACAN ${ }^{1}$, PETRU ADRIAN MIRCEA ${ }^{1}$, \\ LOREDANA BALACESCU ${ }^{2}$, OVIDIU BALACESCU ${ }^{2}$
}

\author{
${ }^{1}$ Department of Internal Medicine, 1st Medical Clinic, Cluj-Napoca, Iuliu \\ Hatieganu University of Medicine and Pharmacy Cluj-Napoca, Romania \\ ${ }^{2}$ Department of Functional Genomics, Proteomics and Experimental Pathology, \\ Prof. Dr. Ion Chiricuta Oncology Institute, Cluj-Napoca, Romania
}

\begin{abstract}
Colorectal cancer is a major cause of cancer-associated deaths in the world. Early detection would be greatly enhanced if accurate and cost-effective diagnostic biomarkers for this disease were accessible. The development of such a blood test will evidently lower the screening costs in regards of colorectal cancer detection. Lately, it has been suggested that microRNA diagnostic biomarkers are feasible new screening methods for colorectal cancer. This review summarizes the diagnostic potential of circulating microRNA biomarkers in relation with colorectal cancer, as well as current methods to detect them.
\end{abstract}

Keywords: colorectal cancer, miRNAs, biomarkers, diagnostic

\section{Introduction}

Colorectal cancer $(\mathrm{CRC})$ is the third most common cancer in the world, especially due to the deficiency of effective biomarkers with sufficient sensitivity and specificity to diagnose CRC at early stages [1]. Colorectal carcinogenesis is a very complex process which implies interactions between tumor and stromal cells, genetic changes and alterations in various molecular signaling pathways $[2,3]$.

Peripheral blood is an accessible body fluid which can be used for non-invasive screening of early CRC. Certainly, an ideal sensitive plasma biomarker which is able to accurately predict the presence of premalignant lesions would have a great clinical application. Implementation of non-invasive biomarkers based on serum profiling could improve the screening tests for CRC. This could be a consistent progress with a great impact on clinical oncology and gastroenterology.

Current screening methods, such as the fecal occult blood test (FOBT), stool DNA test, double contrast barium enema and colonoscopy, present various limitations and/or

Manuscript received: 04.09.2015

Accepted: 18.09.2015

Address for correspondence: roberta786@yahoo.com disadvantages. Colonoscopy is invasive and entails some risks, while the other methods do not have sensitivity. Therefore, there are efforts directed towards developing novel diagnostic screening biomarkers for CRC. New molecular procedures for the detection of CRC are nowadays being analyzed, but most of these still have to be confirmed in large, randomized trials prior to the clinical application. This review presents an overview of the recent reports describing different microRNAs which could be used for the early diagnosis of CRC.

Current methods for detection of miRNAs in the blood samples

In the past few years, researchers have investigated the capability of using microRNAs (miRNAs) as plasma biomarkers for the diagnosis of CRC. MiRNAs are small, 18- to 24-nucleotide RNAs that are involved in several feature of cancer biology, as tumour suppressor genes or oncogenes depending on the cellular conditions in which they are expressed. Several scientific data highlighted the role for miRNAs in CRC initiation, progression and development [4]. However, nowadays challenge is to identify specific biomarkers such as circulating miRNAs, 
and provide supplementary clinical support for CRC diagnosis and prognostic.

Circulating miRNAs can be isolated from serum or plasma, but recent studies have indicated plasma to be a more adequate source for miRNA extraction [5]. However, depending on plasma preparation both the level of circulating miRNA and the accuracy of miRNA biomarkers can be altered. Cheng et al. [6] showed that differences in sample processing regarding filtration and centrifugation modified the levels of residual platelets in samples, which had a significant impact on the level of miRNAs. Consequently, supplementary centrifugation, the refusal of samples with platelet counts above a certain limit and the quantification of hemolysis are mandatory in order to determine the biases that might alter the measuring of miRNA levels.

There are a few studies which have described differences in the quantity and quality of RNA extracted based on the diverse kits used [7-9]. In one of these studies, Kroh et al. [7] analyzed the efficiency of two methods, using extraction kits based on silica-membranebased purification provided by Qiagen and Ambion, and they detected greater miRNA yield following the Qiagen protocol. Moreover, Li et al. [8] carried out an assessment of three commercial kits such as miRNeasy kit (Qiagen), Total RNA Purification Kit (Norgen) and miRVana Kit (Ambion) and discovered that RNA extraction using both Norgen and Qiagen kits have a better quality compared to that obtained by Ambion Kit. Thereby, it is important to pre-evaluate the extraction procedures and to manage the differences of miRNA transcripts that might emerge from various sample preparation, before related them to different pathologies including cancer.

Although several methods to quantify miRNA are currently available, quantitative real-time PCR (qRTPCR) represents the "gold standard" for this approach. Two methods based on TaqMan probes and SYBR Green I labeling are currently used for miRNA detection, and it seems that the TaqMan probes present higher specificity [10].

\section{MiRNAs and colorectal cancer}

Until now, several studies have investigated miRNA expression in CRC and showed that miRNAs are consistently modified in this pathology. An important element of these studies highlighted the higher expression of miRNA in CRC comparing with healthy controls and benign forms, sustaining the hypothesis that abnormal miRNA expression has an important role in CRC initiation and development. After miRNAs discovery, several methods for assessing their relative abundance in biological samples have been developed. Thus, the most used methods include reverse transcription-quantitative PCR (RT-qPCR), miR-microarray hybridization and small RNA sequencing [11].

The capacity to quantify miRNA expression from blood provides a huge advantage as a non-invasive method for CRC diagnosis. MiRNAs represent a promising new tool for the development of CRC biomarkers, because they are surprisingly stable in blood, require less than $2 \mathrm{ml}$ of blood for analysis, and the collected blood can be sent by mail in cold packs (Table I).

Table I. Individual circulating miRNAs as potential diagnostic biomarkers for CRC.

\begin{tabular}{lcc}
\hline miRNA & Findings & References \\
\hline miRNA-21 & Up-regulated in CRC & $\begin{array}{c}\text { Ahmed FE et al [15] } \\
\text { Kanaan Z et al [16] } \\
\text { miRNA-92a }\end{array}$ \\
\hline miRNA-29a & Up-regulated in CRC & Huang et al [13] \\
\hline miRNA-141 & Up-regulated in CRC & Huang et al [13] \\
\hline miRNA-135b & Up-regulated in CRC & Cheng H et al [14] \\
\hline miRNA-95 & Linked with CRC stages & Ng et al [12] \\
\hline miRNA-222 & Up-regulated in CRC & Nagel R et al [23] \\
\hline miRNA-17-3p & Up-regulated in CRC et al [12] \\
\hline miRNA-17-92 cluster & Up-regulated in CRC & Ng et al [12] \\
\hline miRNA-18a & Up-regulated in CRC & Ng et al [12] \\
\hline miRNA-24 & Up-regulated in adenomas & Diosdado B et al [24] \\
\hline miRNA-320a & Up-regulated in advanced adenomas & Giraldez et al [20] \\
\hline miRNA-423-5p & Down-regulated in CRC & Fang et al [18] \\
\hline
\end{tabular}


$\mathrm{Ng}$ et al. [12] described for the first time the quantification of miRNAs in the plasma from CRC patients by real-time qRT-PCR. They have found elevated plasma levels of miRNA-135b, miRNA-95, miRNA-222, miRNA17-3p and miRNA-92 in CRC patients when compared to control group. Furthermore, the elevation of miRNA-92 could distinguish CRC from other diseases, such as gastric cancer and inflammatory bowel disease. Similar results sustained the presence of miRNA-29a and miRNA-92a in CRC plasma and pointed out their role as new possible biomarkers for CRC early screening [13]. In addition, Cheng et al. [14] studied the link between CRC stages and circulating miRNAs levels. They quantified miRNA-21, miRNA-92 and miRNA-141 by qRT-PCR and revealed that only the miRNA-141 level was notably connected with CRC stages, despite the fact that all of the three miRNAs showed different expression in CRC plasma when compared to control groups [14].

Several studies revealed the oncogenic role of miRNA-21 by down-regulating of tumor-suppressor genes and busting of cell proliferation [14, 15]. High levels of plasma miRNA-21 can anticipate the CRC incidence with $90 \%$ specificity and sensitivity [16]. Besides that, miRNA-21 has been found increased in many other solid tumors [17], having an important role in cancer initiation, progression and metastasis. Recently, Fang et al. [18] revealed that plasma levels of miRNA-24, miRNA-320a and miRNA-423-5p have promising potential as novel biomarkers for early detection of CRC, with a sensitivity of $77.78 \%, 90.74 \%$ and $88.89 \%$, respectively. These circulating microRNAs were down-regulated in CRC patients.

Despite the high diagnostic potential of a single miRNA, various studies have focused on the elaboration of a panel of multiple miRNAs to serve as biomarkers (Table II). Kanaan et al [19] carried out a study analyzing 380 plasma miRNAs, and discovered a panel of 8 miRNAs (miRNA- 532-3p, miRNA-331, miRNA-195, miRNA-17, miRNA142-3p, miRNA-15b, miRNA-532 and miRNA-652) that could distinguish patients with adenomas from healthy controls, as well as a panel of 5 miRNAs (miRNA-331, miRNA-15b, miRNA-21, miRNA-142-3p and miRNA339-3p) that could distinguish advanced colorectal adenoma from CRC patients. Giraldez et al [20] also found out a panel of 6 miRNAs (miRNA-18a, miRNA-19a, miRNA19b, miRNA-15b, miRNA-29a and miRNA-335) to be up-regulated in CRC patients, and discovered miRNA18a to be notably up-regulated in patients with advanced adenomas. Although adenomas are benign tumors, part of them may transform into malignant tumors [21, 22]. Some miRNAs involved in tumorigenesis present aberrant expression even since the adenoma stage. MiRNA-21 is a convenient exemplification of this because it is elevated in both adenomas and colon carcinomas [19]. MiRNA-135b may also have a role in the early stages of CRC because it has been found increased in adenomas and one of its targets involve the adenomatous polyposis coli (APC) gene, a constituent of the Wnt signaling pathway commonly downregulated in adenomas [23]. The miRNA-17-92 cluster has also high expression in adenomas and thought it might have a role in the adenoma-carcinoma transition [24]. In a recent study which enrolled 113 patients with CRC, Wang et al [25] evaluated miRNAs panels isolated from serum, and discovered a panel of 6 miRNAs that could accurately detect CRC incidence (miRNA-21, let-7g, miRNA-31, miRNA-92a, miRNA-181b and miRNA-203) with 93\% sensitivity and $91 \%$ specificity, significantly higher than any single conventional biomarker such as CEA or CA199. In the same study, for the same serum samples, the sensitivity of CRC detection by CA19-9 and CEA were $35 \%$ and $23 \%$, respectively. Thereby, circulating miRNAs and especially a combination of multiple serum miRNAs appear to become promising non-invasive biomarkers mainly for early detection of CRC including adenomas.

Table II. Panel of circulating miRNAs as potential diagnostic biomarkers for CRC.

\begin{tabular}{lcc}
\hline miRNA panel & Findings & References \\
\hline $\begin{array}{l}\text { miR-532-3p, miR-331, } \\
\text { miR-195, miR-17, miR-142-3p, } \\
\text { miRw-15b, miR-532, miR-652 }\end{array}$ & $\begin{array}{c}\text { Could distinguish } \\
\text { adenomas from controls }\end{array}$ & Kanaan et al [19] \\
\hline $\begin{array}{l}\text { miRNA-331, miRNA-15b, } \\
\text { miRNA-21, miRNA-142-3p, }\end{array}$ & $\begin{array}{c}\text { Could distinguish } \\
\text { advanced colorectal } \\
\text { miRNA-339-3p }\end{array}$ & Kanaan et al [19] \\
\hline $\begin{array}{l}\text { miRNA-18a, miRNA-19a, } \\
\text { miRNA-19b, miRNA-15b, }\end{array}$ & Giraldez et al [20] \\
miRNA-29a, miRNA-335 & Up-regulated in CRC & \\
\hline $\begin{array}{l}\text { miRNA-21, let-7g, miRNA-31, } \\
\text { miRNA-92a, miRNA-181b, }\end{array}$ & Wang et al [25] \\
miRNA-203 & Up-regulated in CRC & \\
\hline
\end{tabular}




\section{Conclusions}

Clinical studies including significant numbers of CRC patients (covering all the stages of CRC) as well as patients with precancerous lesions (small adenomas and advanced adenomas) and control patients are needed in order to confirm the value of circulating miRNAs in $\mathrm{CRC}$ detection. Although individual miRNA markers are promising tools for the detection of CRC, a panel of miRNA biomarkers could improve the accuracy of early diagnosis of this malignancy. The development of noninvasive biomarkers might determine a higher compliance in CRC screening rates, earlier diagnosis of CRC and in general a decrease in cancer burden.

\section{Acknowledgements}

This paper was published under the frame of European Social Found, Human Resources Development Operational Programme 2007-2013, project no. POSDRU/159/1.5/S/138776.

\section{References}

1. GLOBOCAN 2012. IARC, WHO. Available from: http:// globocan.iarc.fr

2. Grivennikov SI, Karin M. Dangerous liaisons: STAT3 and NFkappaB collaboration and crosstalk in cancer. Cytokine Growth Factor Rev. 2010;21:11-19.

3. Manzat-Saplacan RM, Mircea PA, Balacescu L, Chira RI, Berindan-Neagoe I, Balacescu O. Can we change our microbiome to prevent colorectal cancer development?. Acta Oncol. 2015;54(8):1085-1095.

4. Ma Y, Zhang P, Yang J, Liu Z, Yang Z, Qin H. Candidate microRNA biomarkers in human colorectal cancer: systematic review profiling studies and experimental validation. Int J Cancer. 2012;130(9):2077-2087.

5. Wang K, Yuan Y, Cho JH, McClarty S, Baxter D, Galas DJ. Comparing the MicroRNA spectrum between serum and plasma. PLoS One. 2012;7:e41561.

6. Cheng HH, Yi HS, Kim Y, Kroh EM, Chien JW, Eaton KD, et al. Plasma processing conditions substantially influence circulating microRNA biomarker levels. PLoS One. 2013;8:e64795.

7. Kroh EM, Parkin RK, Mitchell PS, Tewari M. Analysis of circulating microRNA biomarkers in plasma and serum using quantitative reverse transcription-PCR (qRT-PCR). Methods. 2010;50:298-301.

8. Li Y, Kowdley KV. Method for microRNA isolation from clinical serum samples. Anal Biochem. 2012;431:69-75.

9. Monleau M, Bonnel S, Gostan T, Blanchard D, Courgnaud V, Lecellier $\mathrm{CH}$. Comparison of different extraction techniques to profile microRNAs from human sera and peripheral blood mononuclear cells. BMC Genomics. 2014;15:395.

10. Benes V, Castoldi M. Expression profiling of microRNA using real-time quantitative PCR, how to use it and what is available. Methods. 2010;50(4):244-249.

11. Mestdagh P, Hartmann N, Baeriswyl L, Andreasen D, Bernard
$\mathrm{N}$, Chen $\mathrm{C}$, et al. Evaluation of quantitative miRNA expression platforms in the microRNA quality control (miRQC) study. Nat Methods. 2014;11(8):809-815.

12. Ng EK, Chong WW, Jin H, Lam EK, Shin VY, Yu J, et al. Differential expression of microRNAs in plasma of patients with colorectal cancer: a potential marker for colorectal cancer screening. Gut. 2009;58:1375-1381.

13. Huang Z, Huang D, Ni S, Peng Z, Sheng W, Du X. Plasma microRNAs are promising novel biomarkers for early detection of colorectal cancer. Int J Cancer. 2010;127:118-126.

14. Cheng H, Zhang L, Cogdell DE, Zheng H, Schetter AJ, Nykter $\mathrm{M}$, et al. Circulating plasma MiR-141 is a novel biomarker for metastatic colon cancer and predicts poor prognosis. PLoS One. 2011;6:e17745.

15. Ahmed FE, Amed NC, Vos PW, Bonnerup C, Atkins JN, Casey $\mathrm{M}$, et al. Diagnostic microRNA markers to screen for sporadic human colon cancer in blood. Cancer Genomics Proteomics. 2012;9:179-192.

16. Kanaan Z, Rai SN, Eichenberger MR, Roberts H, Keskey B, Pan J, et al. Plasma miR-21: a potential diagnostic marker of colorectal cancer. Ann Surg. 2012;256:544-551.

17. Volinia S, Calin GA, Liu CG, Ambs S, Cimmino A, Petrocca $\mathrm{F}$, et al. A microRNA expression signature of human solid tumors defines cancer gene targets. Proc Natl Acad Sci U S A. 2006;103:2257-2261.

18. Fang Z, Tang J, Bai Y, Lin H, You H, Jin H et al. Plasma levels of microRNA-24, microRNA-320a, and microRNA-423-5p are potential biomarkers for colorectal carcinoma. J Exp Clin Cancer Res. 2015;34(1):86.

19. Kanaan Z, Roberts H, Eichenberger MR, Billeter A, Ocheretner G, Pan J, et al. A plasma microRNA panel for detection of colorectal adenomas: a step toward more precise screening for colorectal cancer. Ann Surg. 2013;258:400-408.

20. Giráldez MD, Lozano JJ, Ramírez G, Hijona E, Bujanda L, Castells A, et al. Circulating microRNAs as biomarkers of colorectal cancer: results from a genome-wide profiling and validation study. Clin Gastroenterol Hepatol. 2013;11:681-688.

21. Bas B, Dinc B, Oymaci E, Mayir B, Gunduz UR. What are the Endoscopic and Pathological Characteristics of Colorectal Polyps?. Asian Pac J Cancer Prev. 2015;16(13):5163-5167.

22. Coleman HG, Loughrey MB, Murray LJ, Johnston BT, Gavin AT, Shrubsole MJ, et al. Colorectal Cancer Risk Following Adenoma Removal: A Large Prospective Population-Based Cohort Study. Cancer Epidemiol Biomarkers Prev. 2015;24(9):13731380.

23. Nagel R, le Sage C, Diosdado B, van der Waal M, Oude Vrielink JA, Bolijn A, et al. Regulation of the adenomatous polyposis coli gene by the miR-135 family in colorectal cancer. Cancer Res. 2008;68:5795-5802.

24. Diosdado B, van deWiel MA, Terhaar Sive Droste JS, Mongera S, Postma C, Meijerink WJ, et al. MiR-17-92 cluster is associated with 13q gain and c-myc expression during colorectal adenoma to adenocarcinoma progression. Br J Cancer. 2009;101:707-714. 25. Wang J, Huang SK, Zhao M, Yang M, Zhong JL, Gu YY, et al. Identification of a circulating microRNA signature for colorectal cancer detection. PLoS One. 2014;9:e87451. 